Erschienen in: Busch, D./Schröder, H. (Hrsg.): Perspektiven interkultureller Mediation. Grundlagen zur kommunikationswissenschaftlichen Analyse triadischer Verständigung. Studien zur interkulturellen Mediation Bd. 2. Frankfurt/Main: Peter Lang - Frankfurt am Main/Berlin/Bern/Bruxelles/New York/Oxford/Wien: Lang, Gesprächsanalyse von Schlichtungs-Interaktion. Methodische Probleme und ihre Hintergründe

Werner Nothdurft und Thomas Spranz-Fogasy

Zuerst erschienen als: Nothdurft, Werner/Spranz-Fogasy, Thomas (1991): "Gesprächsanalyse von Schlichtungs-Interaktion. Methodische Probleme und ihre Hintergründe". In: Flader, Dieter (Hrsg.): Verbale Interaktion. Studien zur Empirie und Methodologie der Pragmatik. Sturtgart: Metzler: 222-240.

\title{
Schlichtung: Eine Arbeitsdefinition
}

Das Schlichten von Streit ist ein kommunikatives Muster, das nach aller Erfahrung in vielen Ausschnitten sozialer Wirklichkeit vorkommt und offenbar fest zum Bestand kommunikativer Praktiken und Bewältigungsformen sozialer Konflikte durch die Gesellschaftsmitglieder gehört. Trotz aller Variabilität und Varianz von Schlichtungs-Manifestationen läßt sich bei erster Betrachtung dieses Gegenstandes eine "Kernstruktur” von Schlichtung angeben, aus der sich eine Arbeitsdefinition für weitergehende Analyse ergibt.

Wir sprechen von Schlichtung dann, wenn in einen laufenden Streitfall, den die Parteien nicht lösen können oder wollen, ein unabhängiger Dritter eingeschaltet ist, um eine Einigung zwischen den Streitparteien zu erzielen.

\section{Forschungsinteressen}

Aus kommunikationstheoretischer Sicht interessieren an dem Gegenstand "Schlichtung":

- seine charakteristischen kommunikativen Merkmale als ein Verfahren zur Lösung sozialer Konflikte;

- Varianten von Schlichtung in ihrem Zusammenhang mit situativen und übergeordneten (institutionellen, biographischen) Randbedingungen;

- das Aufgabenprofil, das sich den einzelnen Beteiligten bei der Abwicklung von Schlichtung stellt;

1 Wir danken den Teilnehmern des Kolloquiums des DFG-Schwerpunkt Verbale Interaktion im Januar 1984 in Seewiesen für ihre vielen konstruktiven Beiträge und wertvollen Anregungen. Die Arbeit an diesem Beitrag entstand 1984 im Rahmen des Projekts "Schlichtung" am IdS, Mannheim, mit Unterstützung der DFG. Ergebnisse dieses Projekts liegen vor v. a. in Nothdurft (1990), Notbdurft (1995) Spranz-Fogasy (1986). 
- die subjektiven Konfliktversionen der Beteiligten und deren Schicksal im Verlauf des Interaktionsgeschehens;

- charakteristische sprachliche Muster und Formeln in Schlichtungsgesprächen.

Die Resultate dieses Analyseprogramms können über die Bestimmung des Handlungsmusters Schlichten hinaus für folgende übergeordnete Forschungszusammenhänge von Interesse sein:

- für Zwecke einer allgemeinen Theorie verbaler Interaktion ergeben sich Aufschlüsse über Besonderheiten rriadischer Kommunikationsmuster (gegenüber dialogischen Handlungsformen), über die Musterhaftigkeit kommunikativen Geschehens und über Struktur und Dynamik von Konsensfindungsprozessen;

- für Zwecke einer Theorie der sozialen Konstruktion von Wirklichkeit ergeben sich Aufschlüsse über die interaktiven Prozesse der Konstitution und Aushandlung sozialer Sachverhalte (hier: Konflikte) und der Aushandlung von Vereinbarungen und Regelungen zukünftigen Handelns;

- für Zwecke einer Theorie sozialer Konflikte ergeben sich Aufschlüsse über die interaktive Dimension von Konflikten und über Verfahren ihrer kommunikativen Bewältigung.

Die bisherige Beschreibung der Projektvorgaben und Ziele zeigt als Hauptaufgabe die wissenschaftlich-deskriptive Darstellung eines Kommunikationstyps. Eine weitere Aufgabe, der wir uns verpflichtet fühlen, die aber auch von professionellen Schlichtern an uns herangetragen wird, ist, aus Ergebnissen des Projekts einen praktischen Anwendungsbezug zu konstruieren. Aus den Analysen können sich sensibilisierende Hinweise sowohl auf das Aufgabenprofil für Schlichtungsteilnehmer als auch auf situative übergeordnete Randbedingungen ergeben. Dafür hilfreich ist die Konzeption analycischer Prädikate als natürlich, d.h. als Prädikate-der-Teilnehmer (siehe unten).

\section{Das Analyse-Instrument: Handlungsschema}

Das begriffliche Instrumentarium zur systematischen analytischen Erfassung von Schlichtungs-interaktionen beruht auf der Annahme von Schlichtung als einem Handlungsschema. Unter einem Handlungsschema wird ein kulturell verbreiteter und von den Gesellschaftsmitgliedern gewußter Vorstellungszusammenhang verstanden, der Angaben über konstitutive Bestandteile der komplexen Handlung enthält („was dazu gehört”), Angaben über die logische Struktur der Handlungsentwicklung („was wann kommt") und Angaben über unerläßliche Beteiligungsvoraussetzungen der Beteiligten („was man dazu braucht”) (Nothdurft (1984a)). Die konstitutiven Bestandteile werden auch als Schemakomponenten bezeichnet, die logische Struktur als Abfolge-Struktur. 
Die Wahl gerade dieses handlungsschematischen Analysekonzeptes für unsere Arbeit begründet sich aus Defiziten analytischer Behandlung von Schlichtung in bisher vorherrschenden Analysedomänen, vor allem in rechtsethnologischen und rechtssoziologischen Untersuchungen. Es lassen sich dort zwei unterschiedliche Analyse-Perspektiven auf Schlichtung feststellen, die aber beide die interaktive Dimension von Schlichtung gerade nicht thematisieren.

Die eine Perspektive läßt sich als paradigmatisch kennzeichnen: Schlichtung erscheint als ein Lösungsverfahren des generellen sozialen Problems der Konfliktbewältigung innerhalb einer Klasse funktionaler Äquivalente mit differentiellen Folgekosten. Im Rahmen dieser funktionalen Betrachtung folgen Leistungszuschreibungen und -vergleich (z.B. Abel (1980)). Die Binnenstruktur von Schlichtung und ihre Dynamik bleiben außerhalb des Fokus analytischer Aufmerksamkeit; nur der von außen gesetzte Erfolg (= Leistung) interessiert.

Die andere Position läßt sich demgegenüber als syntagmatisch kennzeichnen. Schlichtung erscheint als ein Ereignis unter anderen in einer übergreifenden Sequenz unterschiedlicher Anstrengungen zur Bewältigung eines Konflikts (z.B. Nader \& Todd (1978)). Die Binnenstruktur von Schlichtung und ihre Dynamik bleibt auch hier außerhalb des Blickfeldes; allenfalls das Resultat des Prozesses interessiert, weil es den weiteren Bearbeitungsprozeß beeinflußt. Im Fokus analytischen Interesses stehen die übergeordneten sozialen Bedingungen und Beziehungen der Streitbeteiligten.

Beide Ansätze sind in einem weitesten Sinne makroanalytisch. Gegenüber beiden Ansätzen läßt sich der gewählte handlungsschematische Ansatz als mikroanalytisch bestimmen. Für ein genaueres Verständnis von Schlichtung als sozialem Phänomen sind zwar paradigmatische wie syntagmatische Perspektiven wichtig. Für die Erschließung der interaktiven Dimension von Schlichtung ist aber die mikroanalytische Perspektive einschlägig.

Gegenüber anderen interaktionsanalytischen Ansätzen zeichnet sich das handlungschematische Instrumentarium dadurch aus, daß es natürlich, interaktiv und ganzheitlich ist (Nothdurft (1984 b)).

Das Instrument ist natürlich in dem Sinne, daß das interaktive Geschehen aus der Sicht der Beteiligten als Gesellschaftsmitglieder und in alltagsweltlichen Kategorien segmentiert und beschrieben wird. Die Beteiligung an einem komplexen Handlungsschema fordert von den Parteien, daß diese sich wechselweise den Stand der jeweils erreichten Handlungsprogression verdeutlichen, damit eine gemeinsame Orientierung im Handeln geschaffen werden kann. Diese Teilnehmeraktivitäten ermöglichen es dem Analytiker, den Interaktionsverlauf zu rekonstruieren, d.h. in einer Weise nachzuzeichnen, von der berechtigt angenommen werden kann, 
daß sie der Sichtweise entspricht, in der die Teilnehmer selbst das Geschehen betrachtet hatten. Der handlungsschematische Ansatz setzt m. a. W. die Schnitte im Interaktionsgeschehen gerade dort, wo auch die Teilnehmer sie gesetzt bzw. wahrgenommen hatten und beschreibt die segmentierten Passagen unter einer Perspektive, die auch Bestandteil der Sichtweisen der Beteiligten auf das Gespräch gewesen ist.

Das Instrument ist interaktiv in dem Sinne, daß das Geschehen unter dem Gesichtspunkt komplexer interaktiver Größen (Schema-Komponenten) beschrieben wird. Handlungsmuster mit Schema-Charakter sind arbeitsteilig organisiert und kommen in ihren einzelnen Phasen nur durch das konzertierte Zusammenspiel der Interaktionsleistungen der Beteiligten zustande. Interaktionsteilnehmer, die sich in ihrem Handeln an einem komplexen Schema orientieren, stellen diese Arbeitsteiligkeit in Rechnung: Sie tragen zur Herstellung der Komplexe partiell bei und verlassen sich auf korrespondierende Interaktionsleistungen der anderen. Der arbeitsteilige Charakter verleiht handlungsschematischer Interaktion entsprechend eine kooperative Grundstruktur. Der handlungsschematische Ansatz trägt dieser kooperativen Grundstruktur dadurch Rechnung, daß das interaktive Geschehen zu Aktivitätskomplexen zusammengefaßt wird und individuelle Handlungszüge als Beiträge zur Herstellung solcher Komplexe interpretiert und analysiert werden.

Das Instrument ist ganzheitlich in dem Sinne, daß das hypothetisch angenommene Handlungsmuster die analytische Interpretation der einzelnen Aktivitäten bzw. Aktivitätskomplexe leitet. Handlungsschemata sind funktional auf die Erreichung eines bestimmten gesellschaftichen Zwecks bezogen und ausgerichtet. Diese Zwecksetzung beeinflußt das individuelle Handeln der einzelnen und verleiht einzelnen Schema-Komponenten bzw. Aktivitätskomplexen ihren jeweiligen, zweckfunktionalen Charakter. Eine ganzheitliche Interpretation des Geschehens ermöglicht es, diesen zweckfunktionalen Charakter von Handlungskomplexen zu bestimmen und einzelne Aktivitäten auf diese Zwecke hin zu interpretieren.

\section{Das Handlungsschema Schlichtung}

Unter Einsatz dieses Instrumentariums kamen wir bei erster Durchsicht eines Korpus von Schlichtungsgesprächen auf folgende idealtypische Sequenz von Schemakomponenten:

- Definition der Situation

- Etablierung der Parteien

- Bereinigung der Ausgangspositionen

- Sicherung von Einigungsbereitschaft

- Vorschlagsentwicklung 
- Konsensfeststellung

- Auflösung der Situation

\section{Definition der Situation}

In der Definition der Situation etablieren die Teilnehmer die Orientierung am Handlungsschema Schlichtung als Richtschnur für ihr weiteres Verhalten. In institutionellen Kontexten ist die Situationsdefinition durch die Zugangsbedingungen und die Rahmenbedingungen des interaktiven Settings präfixiert.

vergleichsbehörde $5 s 50$ in der sache der frau hannelore beck gegen frau sabine kraft $5 f 5$ uns möscht isch auch vorstelln mein name is kastner -sch bin hier vorsitzender der vergleichsbehörde protokollführerin is frau klarlack $5 \mathrm{fS}$ un herr freistoss is vom inschdidud für deutsche 0 sprache $5 \mathrm{f5} \rightarrow$ äh zur bedeutung der vergleichsbehörde die vergleichsbehörde is also kein gerischt $5 \mathrm{f} 5$ wir können hier also niemanden verurteilen $5 \mathrm{f} 5$ unsere aufgabe die uns der gesetzgeber zugewiesen hat ist also die« zu versuchen in solchen fällen die sisch halt mal dursch das zusammenleben der menschen ereignen 0 äh: hier" ein- nach möglischkeit eine gürlische vorgerischtlische einigung zu finden $5 s 5$ (3001/03)

In informellen Kontexten erfolgt die Definition der Situation oft durch explizite Ankündigungen, charakteristische Signale (Beschwichtigungsgesten) oder auch durch Verdichtung, d. h., das sukzessive Zustandekommen des Schlichtungs-Charakters in einer Sequenz von Handlungszügen (Nothdurft, Schröder \& Spranz-Fogasy (1984)).

\section{Etablierung der Parteien}

In dieser Handlungskomponente erfolgt die Zuweisung der Interaktionsrollen an die Teilnehmer, insbesondere die Selbst- oder Fremd-Zuschreibung der Schlichter-Rolle an einen der Beteiligten. In institutionellen Kontexten ist diese Zuschreibung vorab geregelt, in informellen Zusammenhängen erfolgt sie meist implizit durch Vollzug der Intervention eines Dritten in einen Streit: Durch die Intervention beansprucht der Intervenierende die Schlichter-Rolle für sich.

R: Ja, aber zurück zu' diesem' ernsthaften Anlaß. Ich würd jetzt gerne' Ihnen Gelegenheit geben, also den beteiligten Eltern und ihren Anwälten' Stellung zu nehmen zu den Bausteinen, die wir bisher zusammengetragen haben, ich hab sie ja eingangs schon genannt. Das ist der" der kurze Jugendamtsbericht', die Anhörung der Eltern im Termin im (Herbst) 1981. Dann das Gutachten von Frau P und äh' das, was wir heute von der Zeugin Z ge- 
hört haben. Und nicht zuletzt', sondern als ein wesentlicher Punkt dessen, was ich hier' herausbekommen habe, das, was $T$ gesagt hat. Ich habs eingangs vorgespielt, Herr $A 3$ und Herr A1, äh was er gesagt hat, ich hab auch das Protokoll überreicht' und auch das, we' was er selber nochmal dann $>$ draufgesprochen hat, auf Band. Und' meine Birte ist' die, daß man vielleicht noch ein Mal einen Weg finden kann', für $T$.

$(3003 / 01)[8]$

\section{Bereinigung der Ausgangspositionen}

In dieser Komponente erfolgt eine erste Konstitution des Gegenstands, über den die Schlichtung erfolgen soll. Die Streitparteien stellen ihre Konfliktversionen des umstrittenen Geschehens dar. Die Manifestationen dieser Komponente variieren im Ausmaß und handlungsmäßiger Ausgestaltung sehr stark: die Versionen können knapp gegenübergestellt werden, es kann aber auch zu ausgedehnten Streitsequenzen der Parteien kommen oder zu ausufernden Expansionen der einzelnen Streitversionen.

C: also sie hätten zeugen dafür ne $5 s 5$ gut so frau

C: kraft sie hawwe jetz also ghört 0 was anliegt was hawwe sie dezu

A:

ja

C: zu sagen $5 f 5$

was

A:

äh viele worte haw isch nischt" gsacht $5 f 5$

C: hawwe se=n gsacht fong mer mol mit dem a was se gsacht hawwe

A: debei

A: drecksau haw isch gsacht gut $5 \mathrm{f} 5$ isch hab auch gesagt äh C: $m b m$ ja

A: wenn se se- soll ich=s wortwördich sache was isch gsacht hab $5 \mathrm{fs}$

C: ja bitte bitte bitte

A: des is nämlisch nischtt angegebe un des is der hauptugrund

C: sie brauche sisch do net-

A: was- warum sisch die frau beck geärgert hat $5 \mathrm{f5}$

C: des hat se (3001/03) 


\section{Sicherung von Einigungsbereitschaft}

In dieser Komponente geht es darum, die Bereitschaft der Streitparteien, zu einem Konsens zu kommen, abzuklären und festzuschreiben. Die formale Deklaration dieser Bereitschaft ist wichtig, um gïnstige atmosphärische Voraussetzungen für den nachfolgenden Lösungsversuch des Schlichters zu schaffen (günstiges Verhandlungsklima), die inhaltliche Bestimmung der Bereitschaft ist wichtig als Maßgabe für die Ausgestaltung des Lösungsversuchs.

Im Falle langer Konfliktgeschichte oder hoher Intensität des Streites ist bei der Realisierung dieser Komponente die Erzielung eines positiven Resultats (einigungsbereit) fraglich.

C: $\mathrm{mhm}$ sie wolle also 0 sie wolla- wa- hab

isch sie jetz so rischtisch verstand frau beck daß sie also

jetz hier praktisch zum ausdruck bringen wollen daß sie nischt

C: nischt bereit sind hier sisch zu einigen sondern -

B: $\quad$ nä uff die« art nit

B: mit entschuldigung $5 f 5$ wenn se nit irgendwie äh e strof oder (3001/03)

\section{Vorschlagsentwicklung}

In dieser Komponente erfolgt das Anbieten und die Aushandlung einer Sachverhaltsversion des Streitgegenstandes, die der Schlichter auf der Grundlage der bis dahin erfolgten Schema-Progression für einigungsträchtig hält, und die Entwicklung eines Vorschlags für den zukünftigen Umgang mit dem Streitgegenstand. Auch diese Komponente variiert in ihrer Ausgestaltung beträchtlich: von der unmittelbaren Akzeptanz des Vorschlags bis zu komplizierten Aushandlungssequenzen mit Stufungen, Schleifen, Verfahrensabbrüchen und -Wiederaufnahmen etc.

C: so" $5 \mathrm{~g} 50$ äh isch glaub den vorfall ham=ma jetzt" soweit er aufzuklären war hier" mit meinen bescheidenen mitteln glaub isch habe mer=n aufgeklärt dass also" $5 \mathrm{~g} 50$ sie" sich in de vergangenheit $5 \mathrm{~g} 50$ solang sie zusammen im gleiche haus gewohnt

C: habe net grad bes"tens vertragen haben $5 \mathrm{~g} 5$ könne ma wohl unterstelle

$\mathrm{B}_{2}$ : des war nur d- 
$\longrightarrow$ C: wär es nischt eine möglischkeit $5 \mathrm{~g} 50$ dass man hier sacht $5 \mathrm{f5} 0$ äh gut was passiert ist ist passiert $5 s 50$ wir sind ja beide auseinander $5 s 50$ a: : h $5 \mathrm{~g} 50$ es lässt sisch heute oh"nedies nach so langer zeit kaum noch mit letzter sischerheit aufklären wer" $5 \mathrm{~g} 50$ was" gesacht hat $5 \mathrm{~s} 50 \mathrm{a}: \mathrm{h}$ wir entschuldigen uns: $5 \mathrm{~g} 50$ gegenseitisch $5 \mathrm{~g} 50$ für das was passiert ist $5 \mathrm{~g} 50$ die kostn die hier entschtandn sind $5 \mathrm{~g} 50$ ä: h gut $5 \mathrm{f} 50$ die werdn geteilt u: $\mathrm{n} 5 \mathrm{~g} 50$ in Zukunft verschprechn wir soweit" wir noch irgendwo $5 \mathrm{~g} 50$ äh in berührung miteinander kom"men $5 \mathrm{f5} 0$ äh dass wir uns gegenseitich reschpektiern und in ruhe lassen wär des net $\mathrm{n}$ weg $5 \mathrm{~g} 500$

$(3001 / 01)$

\section{Konsensfeststellung}

In der Konsensfeststellung deklarieren die Parteien die zustandegekommene Einigung. In institutionellen Kontexten bedarf die Konsensfeststellung häufig der schriftlichen Form, aber auch in informellen Kontexten ist sie meist als eigens markierte, herausgehobene und abgesicherte Aktivität zu beobachten.

PA:

so 5 f5 0 die sandra

DO:

SA:

HE: $\quad$ ja $5 f 5$ jetz 0 darf dann darf der erster $5 f 5$

$\mathrm{PA}$ : is jetz schon von der treppe wieder wech $5 \mathrm{~s} 50$ doris dann kannst

DO:

SA:

$$
n \ddot{a} "=\ddot{a}=\ddot{a}
$$

HE:

PA: du" ja springn 55502,70 alles klar $5 s 5$ alles klar $5 s 5$

DO: papi tu mal das polster

SA:

$\mathrm{HE}$ :

$\mathrm{K}$ :

PA: $\quad$ ja $5 \mathrm{~g} 50$ doris $5 \mathrm{f5}$

DO: weg5f5 0 ja $5 f 5$

(3000/01) 


\section{Auflösung der Situation}

In dieser Komponente wird das Schlichtungs-Schema als solches abgeschlossen; es effolgen Honorierungen der Schlichtungsleistung, nochmalige Zustimmungen der Akzeptabilität des Vorschlags, Anerkennung der Vernünftigkeir der Streitparteien und Feststellung, daß der Streitgegenstand nun „aus der Welt sei”.

\section{C: $\quad 0210$ so damit is des verfahre}

K: BEIDE UNTERSCHREIBEN

C: erledischt sie krigge jetz in de nächschten tagen von uns diese 0 äh erklärung un sie kümmern sisch drum daß die frau

C: beck ihr fuffzisch mark kriggt gell net daß die gschischt do da dran

A: ja

C: scheitern sollt $5 \mathrm{f} 5$

B: $\quad$ sin se gut devu kumme 0 känne se unserm herrgott

B: in de kirsch danke $5 f 5$

A: 090 widdersehn (GEHT AB)

C: widdersehn

B: 0140 widdersehn (GEHT AB)

C: widdersehn

\section{Methodische Probleme und ihre Hintergründe}

Der handlungsschematische Ansatz erwies sich bei der empirischen Arbeit an einem Korpus von Schlichtungsgesprächen als brauchbar für die Erstellung einer ersten Grobanalyse des jeweiligen Geschehens. Aber schon in diesem ersten Analysestadium traten bereits eine Reihe methodischer Probleme auf. Aufgrund der Häufigkeit, mit der diese Probleme auftraten und ihrer Zentralität für den handlungsschematischen Ansatz nahmen wir an, daß diesen Problemen bei der handlungsschematischen Analyse von Schlichtung systematische Bedeutung zukommt, d.h. daß sie in irgendeiner Form Merkmale des Gegenstandes selbst widerspiegeln. ${ }^{2}$

${ }^{2}$ Wir halten die Auffassung, daß die fescgestellten Probleme zu einer Aufgabe des handlungsschematischen Ansazzes führen sollten, für vorschnell. Eine solche Auffassung begibt sich der Chance, in inhaltlicher Auseinandersetzung mit den auftretenden Problemen Neues über den untersuchten Gegenstand zu lernen (analog argumentieren auch Jefferson \& Lee (1981)). 
Die Annahme systematischer Bedeutung methodischer Probleme erwies sich forschungstechnisch als ein Erkenntnisinstrument, ein tieferes Verständnis des untersuchten Gegenstandes zu gewinnen und weitere Merkmale von Schlichtung (seine „Kernstruktur”) zu bestimmen.

Die methodischen Probleme mit dem handlungsschematischen Ansatz, denen wir systematischen Charakter zugeschrieben haben, sind:

1. Vagheit der Aktivitärskomplexe

2. Rollenfixiertheit des Handlungsschemas

3. Organisationsvarianz des Handlungsschemas.

\section{Vagheit des handlungsschematischen Charakters von Aktivitätskomplexen}

Bei der empirischen Analyse von Schlichtungsgesprächen zeigte sich, daß sich oft Passagen der Gespräche nicht als homogene, schlichtungs-charakteristische Aktivitätskomplexe bestimmen ließen. Dieser Befund ergab sich vornehmlich dadurch, daß Aktivitäten, die charakteristischerweise einem bestimmten Schemakomplex angehören, mit Aktivitäten vernetzt auftraten, die einem anderen Komplex zugehören, oder daß erkennbar schema-zugehörige Aktivitäten durch die darauf folgenden Handlungszüge von anderen Teilnehmern nicht als solche behandelt wurden.

Im folgenden Beispiel tauchen Aktivitäten der Schemakomponente Sicherung von Einigungsbereitschaft mit Aktivitären der Komponente Vorschlagsentwicklung vernetzt auf.

R: Ist denn so eine äh Vereinbarung für Sie überhaupt denkbar" für Sie, beide Seiten.

AB: Vereinbarung sicher, sicher' ebenso sicher nicht mir Klausel, in der dann drinsteht, Besuchsrecht fällt fort, dafür ist der Weg zu Familiengericht eröffnet, falls etwas passiert, falls man etwa'

$(3003 / 01)^{3}$

Im nächsten Beispiel folgen auf die beginnende Vorschlagsentwicklung durch C Aktivitäten von $B$, mit denen diese ihre Streitversion neuerlich darlegt und sich nicht mit der vorgeschlagenen Lösung auseinandersetzt.

${ }^{3}$ Dieser Gesprächsausschnitt wurde nicht nach den Standards der Transkriptionsvorschriften des IdS verschriftlicht. Kurze Pausen sind hier mit einfachen Anführungszeichen (") markiert. 
C: $\quad$ sch mein passiert is passiert $-s$ läßt sisch ni=mehr ungschehe mache äh isch würde die sache so sehen daß mer se aus der weit bringe wenn sie sisch für diese 0 äh 0 beleidigungen die se da ausgsproche hawwe entschuldigen 0 äh auf der anren seite frau beck muß isch nadierlisch auch ihnen sagen 0 äh 0 es wär vielleisch besser gewesen wenn sie sisch über die kinder aufgeregt hätten wenn se also zu der frau kraft gegangen wär"ren=n hätte gsach frau kraft ibr

B: oh deswar

C: kinder-ihr kinner- ja 3 s ah des is jo a" kä eistellung s3

B: $\quad$ hopfe un malz verlore

C: $\quad$ mer muß doch- mer muß doch-

B: $\quad$ is des schlimm wenn isch zum=e kind sach des derfsch nit

B: $\quad$ mache 0 die- $\mathrm{d}$ - stä an die wäsch schmeiße $5 s 5$ de $=s$ doch kän

B: $\quad$ grund daß die frau- die hätt jo zu mir kumme känne un sache

A:

die klä

B: $\quad$ känne fra beck was war $=n$ do los $5 s 5$

Der Befund nicht homogener, vager, Aktivitätskomplexe widerspricht der handlungsschematischen Annahme, daß sich die Teilnehmer im Rahmen des Handlungsschemas Schlichten wechselseitig verdeutlichen, welche Schema-Komponente sie gerade handlungsmäßig bearbeiten und an welchem Stand der SchemaProgression sie sich gerade befinden. Unter methodischen Gesichtspunkten stellt diese Annahme sicher, daß sich aufgrund der Verdeutlichungsleistungen der Teilnehmer auch aus extrakommunikativer Perspektive der Bearbeitungsstand des Handlungsschemas feststellen läßt. Das methodische Problem, das sich aus dem Widerspruch von Annahme und Befund ergibt, ist, daß die Beschreibung des Schlichrungsgeschehens in terms von Aktivitätskomplexen nicht mehr eindeutig ist.

Das systematische Merkmal von Schlichtung, das der festgestellten Vagheit der Aktivitätskomplexe zugrundeliegen könnte, sehen wir in der für Schlichtung erforderlichen Verhandlungsfähigkeit der Positionen. ${ }^{4}$

Demonstration und Bewahrung von Verhandlungsfähigkeit ist offenbar konstitutives Merkmal von Schlichtungs-interaktion. Es findet sich sowohl in kulturvergleichenden Analysen (Heeschen (mündliche Mitteilung)) wie auch in ontogenetisch frühen Konflikr-Situationen: Schon im Kindergarten ist die Strategie von Kindern zu beobachten, in Aushandlungsprozessen sich nicht auf Positionen festlegen zu lassen (Grammer (1982)), Möglicherweise gehört es zur 
Schlichtung zeichnet sich gegenüber anderen Verfahren der Bearbeitung sozialer Konflikte ja dadurch aus, daß die Beilegung des Konflikts durch Einigung erfolgt, d.h. durch Veränderung von Ausgangspositionen der Streitparteien (und nicht etwa durch Urteil eines Dritten). Die Positionen, insbesondere zu Lösungsvorschlägen, müssen verhandelbar sein. $\mathrm{Zu}$ dieser Verhandlungsfähigkeit von Positionen gehört dazu, daß sie vage gehalten werden, daß Versuchsballons und Sondierungsversuche mit Drohungen und überzogenen Forderungen abwechseln, daß geblufft wird, daß man sich ohne große Manöver von Positionen zurückziehen und ohne Gesichtsverlust dem Ändern entgegenkommen kann. Zu diesem für Aushandlungen typischen Vage-Halten von Positionen gehört offenbar auch dazu, daß man unklar macht, in welchem Stadium der Schema-Progression man sich befindet: ${ }^{5}$

- ob eine Sachverhalts-Position bereits als Lösungsvorschlag zu werten ist oder noch als Vorbedingung von Einigungsbereitschaft;

- ob es sich bei einem Handlungszug im Rahmen der Darstellung von Ausgangspositionen schon um einen Einigungsvorschlag handelt oder noch um einen Aspekt der eigenen Konflikt-Version;

- ob die Zurückweisung eines Vorschlages durch Aufrollen der Fallgeschichte dessen Ablehnung bedeutet oder nur die eigenen Ansprüche noch einmal in Geltung geserzt werden sollen;

- ob durch Feststellung von Dissens der situative Rahmen in Auflösung begriffen ist oder nur das Handlungs-Schema bis zur Grenze ausgereizt wird, bevor eigene Lösungs-Vorstellungen offeriert werden:

C: also sie sin- 0 sin sie mit einverstandn wenn sisch die frau kraft bei ihne entschuldischt daß sie sisch au"ch entschuldische

C: daß mer=n verglei"sch mache oder net $5 f 5$ ja oder nä frau beck B: ja m-

B: es kummr druff a was noch debei- was sie no"ch zu irgendwie- gra"d mit entschuldische bin isch net oiverstanne $5 f 5$

C: $\quad 3 s$ entschuldische un die fufzisch mark koschde üwernemme ja oder

B: sie mußjo schließlisch a"

Entwicklung von Interaktionskompetenz dazu, zu lernen, Situationen offen zu lassen und interaktive Vagheiten zu ertragen.

s Die Rationalität der Vagheit der Aktivitätskomplexe läßt sich auch aus der frühen kommunikationswissenschaftlichen These der sozialen Schutzfunktion des Kommunikationskonfliktes gegenüber dem sozialen Konflikt herleiten (Richter \& Weidmann (1975)). Es steht zu vermuten, daß diese Schuzfunktion gerade wesentlich auf der Vagheit in der interaktioneilen Ausgestaltung des Geschehens beruht. 


\section{C: $n \ddot{a} s 3$}

B: fer de"s was se misch zammeghe: se hot was i"sch ausghalde hab

B: irgendwie gstrorft werre $5 \mathrm{f5}$ wenn sie" des nit mache mu $\beta$ isch $=s$

$\mathrm{C:} \quad$ ä strof kann isch net ausspreche

B: halt do"ch vor=s gerischt bringe $5 \mathrm{f5}$

C: frau beck $5 \mathrm{f5}$ also in Ordnung $5 \mathrm{f5}$

A: $\quad$ also sie will $=s$ nischt $5 \mathrm{f5}$

B: $\quad$ sie känne- sie känne ihre en-

\section{C: KLOPFT DIE AKTE AUF DEN TISCH}

B: en betrag sage den sie dann äh bringt awwer g"nz grad mit erre entschuldischung nach em:e dreivierteljohr ka" $n$ isch desjo net $5 \mathrm{f} 5$

(3001/03)

\section{Die Rollenfixiertheit des Handlungsschemas Schlichten}

Eine Erwartung, die mit dem handlungsschematischen Ansatz eng verknüpft ist, besteht darin, das interaktive Geschehen als konzertierte Aktion der Beteiligten beschreiben zu können. Die Interaktionspartner sind i. d. R. in gleichem Maße am Zustandekommen der Interaktion beteiligt, und die Analyse muß die Gemeinsamkeit der Organisation angemessen, d. h. in interaktiven Prädikaten, darstellen.

In offensichtlichem Kontrast zu unserer Erwartung war einer der ersten empirischen Befunde im Korpus von Schlichtungsgesprächen die ausgesprochen direktive Steuerung des Geschehens durch den Schlichter: ${ }^{6}$

- Der Schlichter fordert die Streitparteien einzeln und nacheinander auf, ihre Darstellung des Konfliktsachverhalts zu geben:

äh familie neumeier (...) des=s=also der tat"bestand um den es hier geht 5 f5 sie ham sisch bisher noch nicht eingelassen un deswegen sind sie also hier $5 f 5 \mathrm{da} \beta$ wir uns mal darüber unterhalten $5 \mathrm{f5}$ (3/8ff).

- Ebenso äußert er einige Zeit später zur gegnerischen Partei:

ja also herr beck es steht jetzt soweit fescht daß was gsacht" worre is nur natürlich die familie neumeier $5 \mathrm{~g} 50$ äh $5 \mathrm{~g} 5$ bringt hier eine andere version als sie gsacht" habe $5 \mathrm{~g} 5$ jetz sind sie mal dran(11/15ff).

${ }^{6}$ Für die Zwecke dieses Abschnittes können wir uns auf einen Gesprächstext beschränken (3001/01); der institutionelie Zusammenhang dieses Textes kann vernachlässigt werden, da dieselben Phänomene sich auch in privaten Kontexten finden - vielleicht nicht ganz so ausgeprägt. 
- Der Schlichter beendet dann auch eine erste Runde der Darstellung der Parteien:

so" $5 \mathrm{~g} 50$ äh isch glaub den Vorfall ham=ma jetzt" soweit er aufzuklären war hier" mit meinen bescheidenen mitteln glaub isch habe mer=n aufgeklärt (14/3-5)

- und leitet über z. B. zu einer Darstellung dessen, was im Falle einer Nichteinigung geschehen könnte:

äh jetzt möscht ich ihnen bei"dn einmal zu üb-folgendes überlegn geben $5 f f$ (16/7-8).

Auch im Verlauf des weiteren Gesprächs sind es die Aktivitäten des Schlichters, die Handlungskomplexe einleiten und abschließen und dadurch die Interaktion strukturieren.

Eine so ausgeprägte Steuerung des Geschehens durch einen Beteiligten allein kann aber nur gelingen, wenn die Interaktionspartner dessen Aktivitäten akzeptieren und sich nach den Direktiven orientieren. In der Tat wird die Dominanz des Schlichters von den Konfliktparteien anerkannt - in einem Ausmaß, daß wir eine Rollenfixiertheit des Handlungsschemas Schlichten selbst annehmen können.

Neben den bisher beschriebenen Organisationsaktivitäten fallen weitere direktive Maßnahmen auf; dies vor allem dort, wo die Interaktion zwischen den Konfliktparteien zu eskalieren droht. In solchen Momenten fordert der Schlichter die Streitenden auf:

jetz tue se mol longsam $5 f 50$ alle beide $5 f 5$ (15/3),

oder er sorgt für eine gerechte Verteilung des Rederechts:

herr beck sie kumme $5 \mathrm{~g} 50$ mir mir mache des so $6 \mathrm{~g} 60$ je"der kommt zu wort bei uns gell $5 s 5$ (10/2f.).

Deeskalation ist eine wichtige, aber sicher nur flankierende Aufgabe des Schlichters. Zentralere Bedeutung für das Interaktionsziel hat die Aufweichung starrer Fronten - auch hier sind Aktivitäten des Schlichters auffällig deutlich und explizit. wär des net en weg (18/4f) ist nicht nur die Aufforderung an beide Streitparteien, einen fallspezifischen Vermittlungsvorschlag zu bedenken, sondern darüber hinaus der Versuch, die Bedingungen kooperativen Handelns abzuklären. Dies wird im folgenden deutlich, wenn der Schlichter nacheinander die Streitparteien fragt:

- welsche voraussetzunge herr beck (18/7)

- wie schrelln sie sisch zu den $5 \mathrm{~g} 50$ bedingungen die der herr $5 \mathrm{~g} 50$ beck schtellt 5f5 (2I/8ff)

Diese Funktion hat auch die folgende Passage: 
C: würde ihne des net auch reische wenn er auch des hie- wenn mir des hier in einer $5 \mathrm{~g} 50$ vergleischsurkunde $5 \mathrm{~g} 50 \mathrm{zu}$ papier bringen $5 \mathrm{~g} 50$ dass er sisch $5 \mathrm{~g} 50$ für die"sn vorfall $5 \mathrm{~g} 50$ wenigschtens dass er einräumt das er hier also $5 \mathrm{~g} 50 \mathrm{zu}$ schnell war und dass er des bedauert $5 \mathrm{f} 50$

A: des geht ins protokoll dass er sisch entschuldischt $5 \mathrm{f} 5$

C: dasser $=s$

A: das geht in protokoll $5 \mathrm{f5}$ C: bedau"ert dass er $=s$ bedauert $j a$ okay ja gut

Die beiden letzten Befunde, Deeskalation und Abklären von Kooperationsbereitschaft, liefern die Interpretamente für eine systematische Erklärung der Rollenfixiertheit des Handlungsschemas Schlichten. Strukturelle Vorgabe für Schlichtungsgespräche sind die unterschiedlichen Kooperations-Relationen zwischen den Beteiligten. Charakteristisch für Schlichtung ist ja gerade, daß die Streitparteien beim Versuch eines gemeinsamen bilateralen Umgangs mit dem anstehenden Konflikt gescheitert sind. Aufgrund der ungenügenden Kooperationsbereitschaft bzw. fähigkeit kann die Organisation der Schlichtungs-Abwicklung nicht ihnen überlassen bleiben. Bei gleichzeitiger unbelasteter Beziehungsstruktur zwischen jeweiliger Partei und Schlichter liegt es nahe, daß diesem die Organisation der SchlichtungsProgression als Rollenmerkmal zugeschrieben wird, bzw. von ihm beansprucht wird. Qua Rollenfixiertheit sind diese Rechte im Handlungsschema Schlichten selbst angelegt.

\section{Organisationsvarianz des Handlungsschemas}

Das dritte methodische Problem mit dem handlungsschematischen Ansatz, dem wir systematischen Stellenwert zugeschrieben haben, war die Bandbreite, in der sich Schlichtung in unserem Korpus manifestierte. Die Organisationsformen der Schlichtungsphänomene variieren sehr stark und die Schema-Prägnanz ist außerordentlich unterschiedlich ausgeprägt:

- Es gibt „Kurz-Schlichtungen”, die nur minimal vom Interaktionskontext ausgegrenzt werden (z B. Befriedungsaktivitäten in Schlichtungsgesprächen selbst);

- es gibr situativ eingebettete Schlichtungs-Verfahren, deren Charakter als Schlichtung sich erst sukzessive konturiert (z. B. wenn während einer Spielsequenz von Kindern Streit entsteht und ein Elternteil schlichtend eingreift, woraufhin das Spiel wieder aufgenommen wird); 
- es gibt verabredete Schlichtungs-Verfahren mit Festlegung von Raum, Uhrzeit, Beteiligten und vorheriger Aktenzustellung (z. B. Verfahren vor der Vergleichsbehörde oder dem Schiedsmann);

- es gibt aber auch schlichtende Aktivitäten, die als solche oft nur aufgrund des Wissens über Kommunikationsgewohnheiten der Beteiligten bestimmbar wären (z.B. Schlichten als Bestandteil eines pathologischen familialen Interaktionssystems).

Gegenüber dieser festgelegten Variation der Deutlichkeit des Schlichtungs-Charakters der Interaktion war ja die mit handlungsschematischen Ansatz verbundene Erwartung gewesen, daß der Charakter des Geschehens als Schlichtung stets deutlich zutage tritt, wenn auch in der Schema-Abwicklung den jeweiligen situativen Kontingenzen Rechnung getragen wird.

Das methodische Problem, das sich aus diesem Befund ergab, war, daß wir oft den Schlichtungs-Charakter des interaktiven Geschehens zwar intuitiv erfassen konnten, mit Hilfe des handlungsschematischen Instrumentariums aber nicht als solchen nachweisen und explizieren konnten.

Dieser Befund war nicht so eindeutig zu interpretieren, wie die ersten beiden. Es scheint aber folgende Überlegung in diesem Zusammenhang plausibel: Möglicherweise resultiert die unterschiedliche Transparenz von Schlichtungs-Aktivitäten aus der situativ abhängigen Legitimität von Schlichrung als Verfahren der Konfliktbewältigung. Schlichrung ist ein Verfahren, dessen Akzeptabilität nicht in jeder Konfliktsituation als abgegolten und gewährleistet vorausgesetzt werden kann. Schlichtung ist mit Machtansprüchen des Schlichters und Einschränkungen der Handlungsautonomie der Streitparteien verbunden und beinhaltet daher Implikationen, die die Verfahrens-Attraktivität für die Streitparteien im Einzelfall verringern können. Daher kann es sich unter bestimmten situativen Bedingungen für die Beteiligten, insbesondere den Schlichter, als handlungsfunktional erweisen, den Charakter des Geschehens als Schlichtung gerade nicht zu verdeutlichen.

Insgesamt stellt sich der Zusammenhang von methodischen Problemen und ihren Hintergründen folgendermaßen dar:

\begin{tabular}{|l|l|l|l|}
\hline empirischer Befund & Erwartung & $\begin{array}{l}\text { methodlsches Prob- } \\
\text { lem }\end{array}$ & $\begin{array}{l}\text { systematischer Cha- } \\
\text { rakter }\end{array}$ \\
\hline $\begin{array}{l}\text { 1. Vagheit der Kompo- } \\
\text { nenten }\end{array}$ & $\begin{array}{l}\text { Verdeutlichung d. } \\
\text { Komponenten }\end{array}$ & $\begin{array}{l}\text { keine eindeutige Be- } \\
\text { schreibung }\end{array}$ & $\begin{array}{l}\text { Verhandlungsfăhigkeit } \\
\text { der Positionen }\end{array}$ \\
\hline $\begin{array}{l}\text { 2. Rollenfixiertheit der } \\
\text { Komponenten }\end{array}$ & $\begin{array}{l}\text { konzertierte Aktion d. } \\
\text { Betelligten }\end{array}$ & $\begin{array}{l}\text { keine interaktive Be- } \\
\text { schreibung }\end{array}$ & $\begin{array}{l}\text { Unterschiedliche Ko- } \\
\text { operationsbereitschaft }\end{array}$ \\
\hline $\begin{array}{l}\text { 3. Organisationsvari- } \\
\text { anz des Schemas }\end{array}$ & Schema-Konstanz & $\begin{array}{l}\text { keine Beschreibung als } \\
\text { Schema }\end{array}$ & $\begin{array}{l}\text { Legitimität d. Verfah- } \\
\text { rens }\end{array}$ \\
\hline
\end{tabular}




\section{Erhebungsprobleme}

Neben den methodisch-inhaltichen Problemen gab es in der Projektarbeit ein weiteres, zunächst eher äußerliches Problem der Datenerhebung: das Problem der Tonbandaufnahme von Schlichtungs-Gesprächen. Ursache dafür ist u.a. die in den letzten Jahren durch die Diskussion um den Datenschutz noch gewachsene Sensibilität der Öffentlichkeit für die Persönlichkeitsrechte.

Im Falle von Schlichtungsgesprächen ist diese Problematik aber noch verschärft. Bei gerichtlichen Verhandlungen (z.B. der Güterermin beim Arbeitsgericht) sind Ton-oder Videoaufzeichnung laut $\$ 169 \mathrm{GVG}^{7}$ nicht zulässig. Eine Aufnahme für wissenschaftliche Zwecke ist bestenfalls über die Zustimmung aller Beteiligten zu erreichen.

Nichtgerichtliche, aber institutionalisierte Verfahren (z.B. die Vergleichsbehörde in Baden-Württemberg) sind darüber hinaus oft durch eigene explizite Verfahrensregeln vor Öffentlichkeit geschützt. ${ }^{8}$ Für die folgenden Überlegungen entscheidend aber war der Eindruck, daß auch bei alltagsweltlichen Schlichtungsgesprächen eine Tendenz der Teilnehmer wahrnehmbar ist, Personen, die nicht unmittelbar in den Konflikt verwickelt sind, auszuschließen. Schlichtungsgespräche sind offensichtlich in besonderer Weise sensibel und gefährdet. Bei dieser Feststellung kann man es bewenden lassen - man kann aber auch überlegen, ob die Abschottung für den Interaktionstyp Schlichten selber Sinn macht, d.h. man kann ihr in ähnlicher Weise einen systematischen Charakter zuschreiben, wie wir das für die methodischen Probleme gezeigt haben.

Dabei werden zwei Beobachtungen wichtig:

- Konfliktsachverhalte sind zwischen den Streitparteien oft stark wertbesetzt; daraus läßt sich die häufig ausgeprägte Emotionalisierung von Schlichtungsgesprächen erklären.

- Konfliktsachverhalte sind mit gegenseitigen, für die Parteien vorher nicht kompatiblen Ansprüchen verbunden; einmal eingenommene Positionen müssen aber noch verhandlungsfähig bleiben.

Beides, die Preisgabe von Emotionen sowie das Abrücken von Positionen ist vor Außenstehenden, aber auch vor Parteigängern problematisch. Die Abschottung ist nicht absolut - Gerichtsverfahren sind sogar oft ausdrücklich für Öffentlichkeit

7 Gerichtsverfassungsgesetz: $\$ 169$ (Offentlichkeit): *1. Die Verhandlung vor dem erkennenden Gericht einschließlich der Verkündung der Urteile und Beschlüsse ist öffentlich. 2. Tonund Fernseh-Rundfunkaufnahmen sowie Ton- und Filmaufnahmen zum Zwecke der öffentlichen Vorführung oder Veröffentlichung ihres Inhalts sind unzulässig."

8 Verordnung des Justizministeriums über das Sühneverfahren in Privarklagesachen (BadenWürttemberg) vom 23.10.1971: $\$ 6$ Die Sühneverhandlung ist nichtöffentlich,* 
zugänglich, dennoch kommt ihr u. E. eine Bedeutung als interaktionsstabilisierender Faktor zu.

\section{Rationalität des Problems oder Rationalisierung der Phänomene?}

Die Überlegungen zu unseren methodischen Problemen haben sicher nicht die Qualität schlüssiger Aussagen; es sind tentative Interpretationen eines auffälligen Sachverhalts. Diese Charakterisierung weist auf ein theoretisches Problem des analytischen Umgangs mit problematischen empirischen Phänomenen hin. Wir haben versucht, die empirischen Befunde, die wir nicht mit unseren Erwartungen in Einklang bringen konnten, im systematischen Zusammenhang von Schlichtung selbst zu interpretieren. Anhand der Phänomene haben wir Hypothesen über Eigenschaften des Handlungsschemas Schlichten formuliert, unter denen diese Phänomene Sinn erhalten. Ein solches interpretatives Verfahren erhellt Gefährdungen sozialwissenschaftlicher Arbeit von zwei Seiten:

- Jeder analytische Ansatz impliziert Erwartungen, die, wenn sie nicht vom empirischen Material durchbrochen werden, sich ungehindert durchsetzen;

- nicht sofort integrierbare empirische Befunde verleiten dazu, sich ihrer durch Rationalisierung (d.h. Zuschreibung einer vermeintlichen systematischen Bedeutung) zu entledigen; damit gewinnen diese Phänomene an Gewicht, das ihnen so nicht zukommt.

Eine endgültige Lösung dieses Skandalons der sozialwissenschaftlichen Arbeit ist derzeit nicht in Sicht. Eine methodologische Maxime des analytischen Vorgehens läßt sich dafür nur annähernd bestimmen: Überprüfe im Einzelfall, ob Du den empirischen Befund nach deinen Erwartungen einrichtest oder ob Du seine Bedeutung überbewertest.

\section{Ein konzeptioneller Lösungsversuch: Die Kernstruktur von Schlichtung}

Die drei methodenkritisch ermittelten Eigenschaften von Schlichtung stellen im Gegensarz zu den festgestellten Schema-Komponenten keine Verlaufscharakteristika von Schlichtung dar, sondern lassen sich eher als Merkmale begreifen, die offenbar zur Kernstruktur von Schlichtung als einem gesellschaftlich entwickelten und konservierten Verfahren gehören. Als Bestandteile der Kernstruktur sind die Merkmale in ihrer Handlungsrelevanz auch nicht abhängig von der zeitlichen Progression des Schlichtungs-Geschehens, sondern erscheinen dieser gegenüber als konstante Merkmale von Schlichten, deren Relevanzausprägung durch die Einbindung des Schlichtungs-Geschehens in übergeordnete, soziale Handlungszusammenhänge gesteuert wird. 
Diese Anforderungen unter methodischen Gesichtspunkten angemessen in Rechnung zu stellen, macht es erforderlich, das prozeß-sensitive Konzept des Handlungsschemas von Schlichtung, das den Gang der Handlungsprogression und das interaktive Abarbeiten der Schema-Komponenten erfaßt, zu ergänzen durch ein Profil von Anforderungen der Kernstruktur von Schlichtung, das SchlichtungsTeilnehmer in ihrem Handeln in Rechnung stellen müssen. Unter dieser Perspektive stellen sich die methodenkritisch aufgedeckten Phänomene als Resultat simultaner Bewältigung des Anforderungsprofils und des Handlungsschemas dar.

Man kann sich die so ergänzte analytische Konstruktion als "Gitter" vorstellen: Die eine Dimension gibt die Progression des Handlungs-Musters in idealtypischer Weise vor und erlaubt die Segmentierung des Geschehens und Bestimmung von Aktivitätskomplexen (Handlungsschema-Aralyse). Die andere Dimension beinhaltet eine Liste von Eigenschaften der Kernstruktur der Handlungsform, die als interaktive Anforderungen begriffen werden und kontinuierlich von den Interaktionsteilnehmern in ihrem Handeln bearbeitet werden müssen. Die Ausprägung der Anforderungen (ihre Handlungsrelevanz) variiert lokal und situativ kontingent.

Im Einzelfall kann die Bearbeitung einer dieser Anforderungen an einer Gesprächs-Position so ausgebaut sein, daß sie als Manifestation einer Schema-Komponente erscheint. Erst im Fall-Vergleich kann sich dann herausstellen, daß es sich stattdessen um eine ausgebaute Bearbeitung einer Eigenschaft der Kernstruktur handelt. ${ }^{9}$

Eine solche analytische Konstruktion ermöglicht es, über Gesichtspunkte der verlaufsmäßigen Ordnung von Schlichtung hinaus weitere Ordnungsgesichtspunkte für vorfindliche Handlungszusammenhänge oder einzelne Gesprächs-Phänomene in Schlichtungsgesprächen anzugeben und dadurch zu einem genaueren Verständnis des Schlichtungs-Geschehens zu kommen.

Aus der Liste methodenkritisch ermittelter Eigenschaften von Schlichtung sowie anderen Beobachtungen am Datenmaterial ergibr sich folgendes Anforderungsprofil der Kernstruktur für Schlichtung: ${ }^{10}$

- Aufrechterhalten der Neutralität des Schlichters

- Einhalten von Friedenspflichr

- Sicherstellen von Einigungsbereitschaft

' Ein solches Konzept bietet so auch ein Korrektiv gegen die Fehlertendenz, institutioneil ausgeformte Typen eines Handlungsmusters mit diesem selbst zu identifizieren, und ermöglicht es, zwischen sozialgeschichtlich kontingenten Varianten und der kommunikativen Kernstruktur besser unterscheiden zu können.

${ }^{10}$ Diese Kernstruktur wird genauer beschrieben in Nothdurf (1995). 
- Umgehen mit der Muster-Prägnanz des Geschehens

- Sicherstellen von Verhandlungsfähigkeit

- Schaffen von Gerechtigkeit

- Sorgen für Perspektiventransfer.

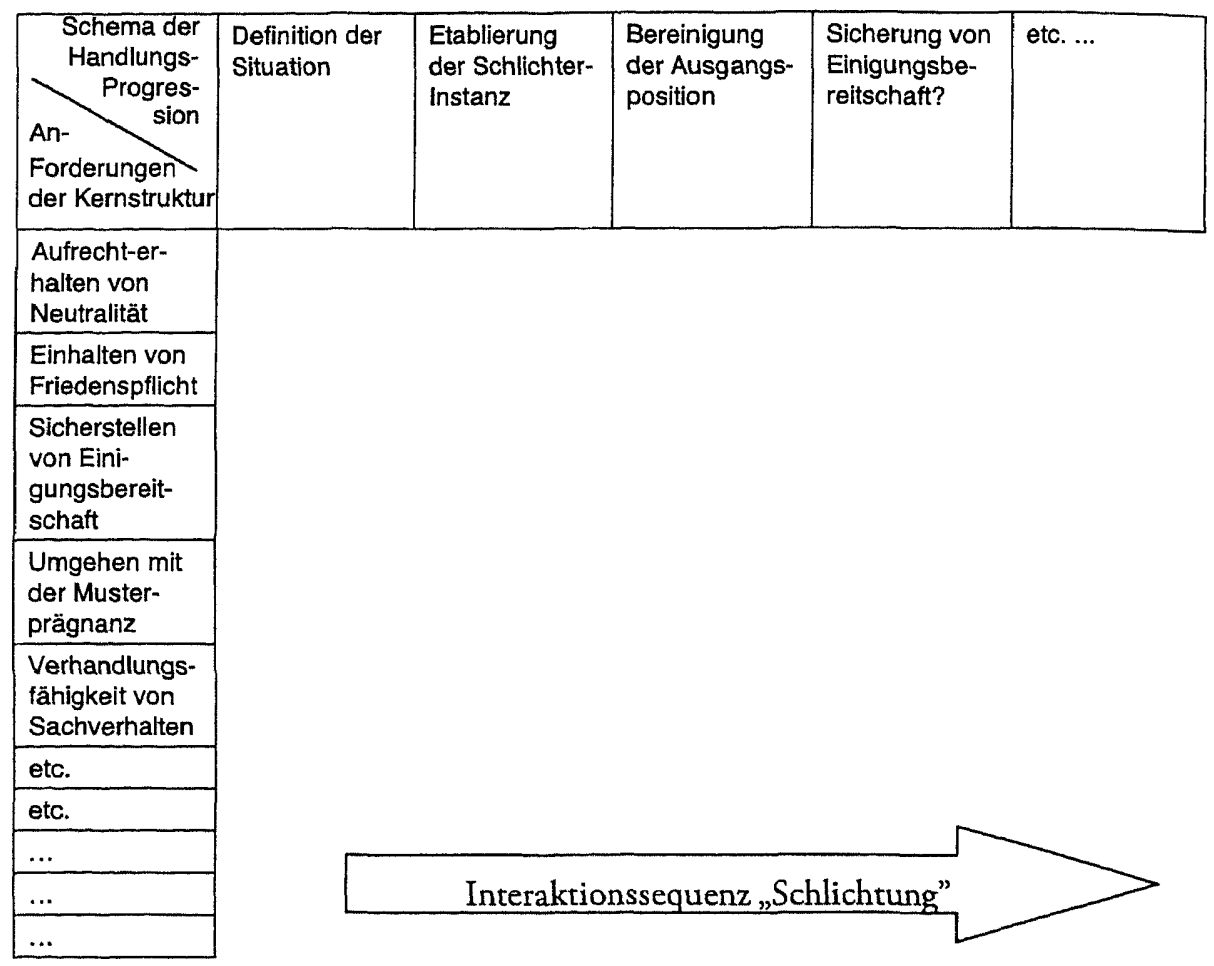

Legende zu den verwendeten Transkriptionszeichen

A : $\quad$ Streitpartei A

B : Streitpartei B

C: Schlichter

$=\quad$ Verschleifung

4f f4 fremdsprachliche Sequenzen

6a a6 Abkürzungen und Buchstabieren

* * Extension der Gültigkeit eines Kommentars 


\begin{tabular}{|c|c|}
\hline- & Wortfragment \\
\hline 11 & Alternativlautung \\
\hline$\ldots$ & unverständliche Sequenzen \\
\hline 0 & kurze Pause \\
\hline 00 & mittellange Pause \\
\hline 03,50 & längere Pause mit Angabe der Dauer in Sekunden \\
\hline $21 \quad 12$ & langsamer \\
\hline $2 s \quad s 2$ & schneller \\
\hline $3 s \quad s 3$ & lauter \\
\hline 3113 & leiser \\
\hline $5 s 5$ & steigende Intonation \\
\hline $5 f 5$ & fallende Intonation \\
\hline $5 g 5$ & gleichbleibende/schwebende Intonation \\
\hline$"$ & nach einer Silbe oder einem Wort: Emphase \\
\hline : & Dehnung \\
\hline
\end{tabular}

\section{Literaturverzeichnis}

Abel, Richard L. (1980): "Theories of Litigation in Sociery". In: Blankenburg, E. et al. (Hrsg.): Alternative Rechtsformen und Alternativen zum Recht. Opladen: 165-191.

Grammer, Karl (1982): Wettbewerb und Kooperation. Strategien des Eingriffs in Konflikte unter Kindern einer Kindergartengruppe. (Ms.).

Jefferson, Gail/Lee, John R. E. (1981): The Analysis of Conversations in Which 'Troubles' and 'Anxieties' are Expressed. Final Report (Ms.).

Nader, Laura/Todd, Harry F. jr. (1978): "Introduction". In: Nader, Laura/Todd, Harry F. (Hrsg.): The Disputing Process. Law in Ten Societies. New York: 1-40.

Nothdurf, Werner (1984a): "...äb folgendes problem äb" Die interaktive Ausarbeitung "des Problems" in Beratungsgesprächen. Tübingen.

Nothdurf, Werner (1984b): "Ein Instrument zur Verlaufsanalyse von Sprechstundeninteraktion". In: Tewes, Uwe (Hrsg.): Angewandte Medizinpsychologie. Bd. 1. Frankfurt/Main: 114-125.

Nothdurft (1990): Konfliktstoff. Berlin.

Nothdurft, Werner (1995): "Gesprächsanalyse von Schlichrung. Die Geschichte eines Forschungsprojekrs und die Entwicklung seiner Ergebnisse". In: Nothdurf, Werner (Hrsg.): Streit schlichten. Gesprächsanalytische Untersuchungen zal institutionellen Formen konsensueller Konfizkrregelung. Berlin/New York: de Gruyter: 1-26.

Nothdurft, Werner / Schröder, Peter / Spranz-Fogasy, Thomas (1984): , Verdichtung'. Das situative Zustandekommen von Schlichrung und Ansätze zur methodischen Erfassung. In: Schlig 2. Mannheim. 
Richter, Helmut/Weidmann, Fred (1978): Semantisch bedingte Kommunikationskonflikte bei Gleichsprachigen. Hamburg.

Spranz-Fogasy, Thomas (1986): 'Widersprechen': Zu Form und Funktion eines Aktivitätstyps in Schlichtungsgesprächen; eine gesprächsanalytische Untersuchung. Vol. 62, Forschungsberichte des Instituts für deutsche Sprache, Mannheim. Tübingen. 